\title{
Matrix form of Legendre polynomials for solving linear integro-differential equations of high order
}

\begin{abstract}
This paper presents an effective approximate solution of high order of Fredholm-Volterra integro-differential equations (FVIDEs) with boundary condition. Legendre truncated series is used as a basis functions to estimate the unknown function. Matrix operation of Legendre polynomials is used to transform FVIDEs with boundary conditions into matrix equation of Fredholm-Volterra type. Gauss Legendre quadrature formula and collocation method are applied to transfer the matrix equation into system of linear algebraic equations. The latter equation is solved by Gauss elimination method. The accuracy and validity of this method are discussed by solving two numerical examples and comparisons with wavelet and methods.
\end{abstract}

Keyword: High order of Fredholm-Volterra integro-differential equations; Boundary condition; Legendre polynomials 